\title{
PRECOMPLETE CLONES ON INFINITE SETS WHICH ARE CLOSED UNDER CONJUGATION
}

\author{
MICHAEL PINSKER
}

\begin{abstract}
We show that on an infinite set, there exist no other precomplete clones closed under conjugation except those which contain all permutations. Since on base sets of some infinite cardinalities, in particular on countably infinite ones, the precomplete clones containing the permutations have been determined, this yields a complete list of the precomplete conjugation-closed clones in those cases. In addition, we show that there exist no precomplete submonoids of the full transformation monoid which are closed under conjugation except those which contain the permutations; the monoids of the latter kind are known.
\end{abstract}

\section{BACKGROUND AND THE RESUlT}

Let $X$ be a set and denote for all $n \geq 1$ the set of $n$-ary operations on $X$ by $\mathscr{O}^{(n)}$. The union $\mathscr{O}=\bigcup_{n \geq 1} \mathscr{O}^{(n)}$ is the set of all operations on $X$ of finite arity. A clone is a subset of $\mathscr{O}$ which contains all projections, i.e. all functions of the form $\pi_{k}^{n}\left(x_{1}, \ldots, x_{n}\right)=x_{k}(1 \leq k \leq n)$, and which is closed under composition of functions. Ordering the clones on $X$ by set-theoretical inclusion, one obtains a complete algebraic lattice $C l(X)$. We are interested in the structure of this lattice for infinite $X$, in which case it has cardinality $2^{2^{|X|}}$.

We call a clone precomplete or maximal iff it is a dual atom in $C l(X)$. The number of precomplete clones on an infinite base set equals the size of the whole clone lattice (15]), and there is little hope to determine all of them. However, the precomplete clones which contain $\mathscr{O}^{(1)}$ have been determined on some infinite $X$ ([2], [5]).

Theorem 1. If $X$ is countably infinite or of weakly compact cardinality, then there are exactly two precomplete clones $\operatorname{Pol}\left(T_{1}\right)$ and $\operatorname{Pol}\left(T_{2}\right)$ above $\mathscr{O}^{(1)}$.

For most other cardinalities of $X$, the number of precomplete clones above $\mathscr{O}^{(1)}$ is $2^{2^{|X|}}$, so in those cases it seems impossible to find them all ([5]).

The precomplete clones which contain the set of permutations $\mathscr{S}$ of the base set but not $\mathscr{O}^{(1)}$ have been determined on countably infinite $X$ in [6], and we extended this result to all $X$ of regular cardinality in [13]. To describe these clones, the following concept was used: For a submonoid $\mathscr{G} \subseteq \mathscr{O}^{(1)}$, define the clone of

1991 Mathematics Subject Classification. Primary 08A40; secondary 08A05.

Key words and phrases. clone lattice, permutations, precomplete clones, conjugation, symmetric clones, unary clones, precomplete monoids.

The author would like to thank L. Heindorf for his remarks on an earlier version of the paper. Support through DOC [Doctoral Scholarship Programme of the Austrian Academy of Sciences], and later through the Postdoctoral Fellowship of the Japan Society for the Promotion of Science (JSPS) is gratefully acknowledged. 
polymorphisms $\operatorname{Pol}(\mathscr{G})$ to consist of all $f \in \mathscr{O}$ satisfying $f\left(g_{1}, \ldots, g_{n}\right) \in \mathscr{G}$ whenever $g_{1}, \ldots, g_{n} \in \mathscr{G}$, where $n \geq 1$ is the arity of $f$. Call a subset $S \subseteq X$ large iff $|S|=|X|$, and small otherwise; $S$ is co-large (co-small) iff its complement is large (small). A property holds for almost all $x \in X$ iff there is a co-small $S \subseteq X$ such that the property holds for all $x \in S$. A function $f \in \mathscr{O}^{(1)}$ is almost surjective iff almost all $x \in X$ are in the range of $f$. Then we have

Theorem 2. Let $X$ be a set of regular cardinality $\kappa$. The precomplete clones over $X$ which contain all bijections but not all unary functions are exactly those of the form $\operatorname{Pol}(\mathscr{G})$, where $\mathscr{G} \in\{\mathscr{A}, \mathscr{B}, \mathscr{E}, \mathscr{F}\} \cup\left\{\mathscr{G}_{\lambda}: 1 \leq \lambda \leq \kappa, \lambda\right.$ a cardinal $\}$ is one of the following submonoids of $\mathscr{O}^{(1)}$ :

(1) $\mathscr{A}=\left\{f \in \mathscr{O}^{(1)}: f^{-1}[\{y\}]\right.$ is small for almost all $\left.y \in X\right\}$

(2) $\mathscr{B}=\left\{f \in \mathscr{O}^{(1)}: f^{-1}[\{y\}]\right.$ is small for all $\left.y \in X\right\}$

(3) $\mathscr{E}=\left\{f \in \mathscr{O}^{(1)}: f\right.$ is almost surjective $\}$

(4) $\mathscr{F}=\left\{f \in \mathscr{O}^{(1)}: f\right.$ is almost surjective or constant $\}$

(5) $\mathscr{G}_{\lambda}=\left\{f \in \mathscr{O}^{(1)}:\right.$ if $A \subseteq X$ has cardinality $\lambda$ then $\left.|X \backslash f[X \backslash A]| \geq \lambda\right\}$

Clones containing the permutations $\mathscr{S}$ have the property that they are closed under conjugation, that is, $\mathscr{C}=\left\{\gamma^{-1}\left(f\left(\gamma\left(x_{1}\right), \ldots, \gamma\left(x_{n}\right)\right)\right): f \in \mathscr{C}, \gamma \in \mathscr{S}\right\}$. We call clones with this property symmetric; they are interesting because they are independent of the order or, indeed, any other structure that one might associate with the base set. But a clone need not contain $\mathscr{S}$ in order to be symmetric: For example, the clone consisting only of the projections and the constant functions is conjugation-closed.

The set of symmetric clones is a sublattice $C l_{\text {sym }}(X)$ of the clone lattice. Whereas $\mathrm{Cl}(\mathrm{X})$ need not be dually atomic [3], that is, not every nontrivial clone is contained in a precomplete one, the sublattice of symmetric clones is. This is because there exist finitely many functions such that the only symmetric clone containing those functions is $\mathscr{O}$ : If $\alpha \in \mathscr{S}$ is a permutation of $X$ which has large support (i.e., if $\{x \in X: \alpha(x) \neq x\}$ is large), then $\alpha$ together with its conjugates (that is, all functions of the form $\gamma^{-1} \circ \alpha \circ \gamma$, where $\gamma \in \mathscr{S}$ ) generate $\mathscr{S}$ (16 for the countable and 1 for the uncountable case). And it is well-known that $\mathscr{O}$ is finitely generated over $\mathscr{S}$ (see for example 6] for countably infinite and 13 for arbitrary infinite $X$ ).

In the light of Theorems 1 and 2] it is natural to ask whether it is possible to obtain a list of all symmetric precomplete clones not containing $\mathscr{S}$. We give the answer to this in this article.

Theorem 3. Let $X$ be infinite. If $\mathscr{C}$ is a symmetric precomplete clone, then it contains all permutations.

We emphasize that this theorem is about symmetric clones which are dual atoms in $C l(X)$, and not about symmetric clones which are dual atoms in the sublattice $C l_{\text {sym }}(X)$ of symmetric clones. There exist clones of the latter type which do not contain all permutations: For example, the clone consisting of all $f \in \mathscr{O}$ for which the set $\{x \in X: f(x, \ldots, x)=x\}$ is co-small does not contain $\mathscr{S}$, is obviously symmetric, and it follows easily from the complete description of the clone lattice above this clone in 4 that there is no non-trivial symmetric clone containing it.

Corollary 4. If $X$ has regular cardinality, then the symmetric precomplete clones which do not contain $\mathscr{O}^{(1)}$ are exactly those from Theorem $\mathbf{Q}$. If $X$ is countably infinite or of weakly compact cardinality, then the symmetric precomplete clones are exactly those from Theorems 1 and . 
A unary clone is a clone consisting only of essentially unary functions, where by an essentially unary function we mean one which depends on only one of its variables. Clearly, though formally different, unary clones can be seen as submonoids of the full transformation monoid $\mathscr{O}^{(1)}$ and we shall not distinguish between the two notions. A precomplete unary clone is a dual atom in the lattice of submonoids of $\mathscr{O}^{(1)}$. In 2], all precomplete unary clones which contain all permutations were determined on countably infinite $X$. The result was generalized in [13 to arbitrary infinite sets: For a cardinal $1 \leq \lambda \leq|X|$ we call a function $f \in \mathscr{O}^{(1)} \lambda$-injective iff there exists $A \subseteq X$ with $|A|<\lambda$ such that the restriction of $f$ to $X \backslash A$ is injective.

Theorem 5. Let $X$ be a set of infinite cardinality $\kappa$. If $\kappa$ is regular, then the precomplete submonoids of $\mathscr{O}^{(1)}$ which contain the permutations are exactly the monoid $\mathscr{A}$ and the monoids $\mathscr{G}_{\lambda}$ and $\mathscr{M}_{\lambda}$ for $\lambda=1$ and $\aleph_{0} \leq \lambda \leq \kappa, \lambda$ a cardinal, where

- $\mathscr{A}=\left\{f \in \mathscr{O}^{(1)}: f^{-1}[\{y\}]\right.$ is small for almost all $\left.y \in X\right\}$

- $\mathscr{G}_{\lambda}=\left\{f \in \mathscr{O}^{(1)}\right.$ : if $A \subseteq X$ has cardinality $\lambda$ then $\left.|X \backslash f[X \backslash A]| \geq \lambda\right\}$

- $\mathscr{M}_{\lambda}=\left\{f \in \mathscr{O}^{(1)}: f\right.$ is $\lambda$-surjective or not $\lambda$-injective $\}$

If $\kappa$ is singular, then the same is true with the monoid $\mathscr{A}$ replaced by

$$
\mathscr{A}^{\prime}=\left\{f \in \mathscr{O}^{(1)}: \exists \lambda<\kappa\left(\left|f^{-1}[\{x\}]\right| \leq \lambda \text { for almost all } x \in X\right)\right\} .
$$

We shall obtain the following result.

Theorem 6. Let $X$ be infinite. The symmetric precomplete unary clones are exactly those of Theorem 5 .

It might be interesting to note that on finite $X$, all symmetric clones are known ([7], [8], [9], [12], [1], see also the survey paper [17]). If $X$ has at least five elements, then the only symmetric precomplete clone is the Stupecki clone of all functions which are either essentially unary or take at most $|X|-1$ values. In this case the clone of all idempotent functions is the only other clone which is maximal in $C l_{\text {sym }}(X)$ (but not in $C l(X)$, since it is properly contained in the clone of all $f \in \mathscr{O}$ for which $f(a, \ldots, a)=a$, for any fixed $a \in X)$. For $|X|<5$ the situation is more complicated, see [17. On all finite $X$ with at least three elements, there exist precisely two symmetric precomplete submonoids of $\mathscr{O}^{(1)}$ : The first one contains all permutations, and is the monoid of all functions $f \in \mathscr{O}^{(1)}$ which are either a permutation or for which the set $\{x \in X: \exists y \neq x: f(x)=f(y)\}$ has at least three elements. The second one does not contain the permutations and consists of the non-permutations plus all even permutations. That there are no other symmetric precomplete monoids, even no other dual atoms in the lattice of symmetric monoids, is not difficult to prove from the definitions of these two monoids and the fact (10]) that if $\mathscr{M}$ is a symmetric monoid on a finite set $X$, then $\mathscr{M} \cup \mathscr{S}$ is a monoid which contains $\mathscr{M}$.

Since on an infinite set $X$ of size $\kappa=\aleph_{\alpha}$ there exist $\max \left\{2^{2^{|\alpha|}}, 2^{2^{\aleph_{0}}}\right\}$ submonoids of $\mathscr{O}^{(1)}$ containing $\mathscr{S}([14])$, describing all symmetric monoids or even clones seems to be hopeless.

1.1. Notation. For a set of functions $\mathscr{H} \subseteq \mathscr{O}$, we use the common notation $\langle\mathscr{H}\rangle$ for the smallest clone containing $\mathscr{H}$. If $n \geq 1$, then $\mathscr{H}^{(n)}$ denotes the $n$-ary functions in $\mathscr{H}$. In particular, if $\mathscr{H} \subseteq \mathscr{O}^{(1)}$ is a set of unary functions, then $\langle\mathscr{H}\rangle^{(1)}$ is the unary part of the clone generated by $\mathscr{H}$ and therefore nothing else 
than the monoid generated by $\mathscr{H}$.

The unary projection $\pi_{1}^{1}$ is the identity function on $X$ and we denote it also by id. For $S \subseteq X$ we denote the image of $S$ under $f$ by $f[S]$.

\section{UnARY ClONES: The Proof of Theorem 6]}

This section contains the proof of the simple unary case (Theorem 6). We start by citing a theorem which is essential for the whole paper; for us, its most important implication is that a symmetric clone which does not contain all permutations in fact contains almost no permutations.

Theorem 7 (16, (1]). If $\alpha \in \mathscr{S}$ has large support, then $\alpha$ together with its conjugates generate $\mathscr{S}$.

Corollary 8. If $\mathscr{C}$ is a symmetric clone which does not contain all permutations, then $\mathscr{C}$ does not contain any permutation with large support.

Lemma 9. If $\mathscr{G}$ is a symmetric submonoid of $\mathscr{O}^{(1)}$ and $\alpha \in \mathscr{S}$, then $\langle\{\alpha\} \cup \mathscr{G}\rangle^{(1)}=$ $\left\{\alpha^{n} \circ g: g \in \mathscr{G}, n \geq 0\right\}=\left\{g \circ \alpha^{n}: g \in \mathscr{G}, n \geq 0\right\}$.

Proof. We begin with the first equality. It is clear that $\left\{\alpha^{n} \circ g: g \in \mathscr{G}, n \geq\right.$ $0\} \subseteq\langle\{\alpha\} \cup \mathscr{G}\rangle^{(1)}$. The other inclusion we prove by induction over terms $t$ in $\langle\{\alpha\} \cup \mathscr{G}\rangle^{(1)}$. The statement is obvious for $t=\alpha$ and $t=g \in \mathscr{G}$. So assume that $t=\alpha \circ s$, where $s \in\langle\{\alpha\} \cup \mathscr{G}\rangle^{(1)}$ satisfies the induction hypothesis. Then there exist $n \geq 0$ and $g \in \mathscr{G}$ with $s=\alpha^{n} \circ g$ so that we have $t=\alpha^{n+1} \circ g$. To finish the induction, assume that $t=h \circ s$, with $h \in \mathscr{G}$ and $s \in\langle\{\alpha\} \cup \mathscr{G}\rangle^{(1)}$ satisfying the induction hypothesis, so that $s=\alpha^{n} \circ g$ for some $n \geq 0$ and some $g \in \mathscr{G}$. Set $h^{\prime}=\alpha^{-n} \circ h \circ \alpha^{n}$. Then $h^{\prime} \in \mathscr{G}$ because $\mathscr{G}$ is symmetric, and $h=\alpha^{n} \circ h^{\prime} \circ \alpha^{-n}$. Hence, $t=h \circ \alpha^{n} \circ s=\alpha^{n} \circ h^{\prime} \circ \alpha^{-n} \circ \alpha^{n} \circ g=\alpha^{n} \circ\left(h^{\prime} \circ g\right)$ and we are finished.

Now for the second equality, observe that $t=\alpha^{n} \circ g$, with $n \geq 0$ and $g \in \mathscr{G}$, if and only if $t=g^{\prime} \circ \alpha^{n}$, where $g^{\prime}=\alpha^{n} \circ g \circ \alpha^{-n} \in \mathscr{G}$.

Proof of Theorem [6] We show that if $\mathscr{G}$ is a symmetric submonoid of $\mathscr{O}^{(1)}$, and if $\mathscr{G} \nsupseteq \mathscr{S}$, then $\mathscr{G}$ is not precomplete. Take $\alpha \in \mathscr{S}$ with large and co-large support, and take $\beta \in \mathscr{S}$ with large and co-small support. Having large support, neither $\alpha$ nor $\beta$ are elements of $\mathscr{G}$, by Corollary 8 , If $\mathscr{G}$ was a precomplete monoid, then every function in $\mathscr{O}^{(1)}$ would be an element of $\langle\{\alpha\} \cup \mathscr{G}\rangle^{(1)}$, which equals $\left\{\alpha^{n} \circ g: g \in \mathscr{G}, n \geq 0\right\}$ by Lemma 9 In particular, there would exist $n \geq 1$ and $\gamma \in \mathscr{G}$ such that $\beta=\alpha^{n} \circ \gamma$. Obviously, $\gamma$ has to be a permutation; being an element of $\mathscr{G}$, by Corollary 8 it must have small support. The power $\alpha^{n}$ still has co-large support, and so does $\alpha^{n} \circ \gamma$. Hence, $\beta$ cannot equal $\alpha^{n} \circ \gamma$, so that we end up with a contradiction.

\section{Non-unary clones: The proof of Theorem 3}

We turn to the proof of Theorem 3 The reason why this is more difficult is that with non-unary functions, Lemma 9 becomes more complicated.

Lemma 10. If $\mathscr{C}$ is a symmetric clone and $\alpha \in \mathscr{S}$ and $t \in \mathscr{O}^{(n)}$, then $t \in\langle\{\alpha\} \cup \mathscr{C}\rangle$ iff there exists some integer $k \geq 1, f \in \mathscr{C}^{(n \cdot k)}$, and a sequence $\left(a_{i, j}: 1 \leq i \leq n, 1 \leq\right.$ $j \leq k$ ) of nonnegative integers such that

$$
t\left(x_{1}, \ldots, x_{n}\right)=f\left(\alpha^{a_{1,1}}\left(x_{1}\right), \ldots, \alpha^{a_{1, k}}\left(x_{1}\right), \ldots, \alpha^{a_{n, 1}}\left(x_{n}\right), \ldots, \alpha^{a_{n, k}}\left(x_{n}\right)\right) .
$$


Proof. It is clear that if $t$ is of that form, then $t \in\langle\{\alpha\} \cup \mathscr{C}\rangle$. The other direction we prove by induction over terms $t$ in $\langle\{\alpha\} \cup \mathscr{C}\rangle$. The statement is obvious for $t=\alpha$ and $t=f \in \mathscr{C}$. So assume that $t=\alpha \circ s$, where $s \in\langle\{\alpha\} \cup \mathscr{C}\rangle$ satisfies the induction hypothesis. Then $s=f\left(\alpha^{a_{1,1}}\left(x_{1}\right), \ldots, \alpha^{a_{1, k}}\left(x_{1}\right), \ldots, \alpha^{a_{n, 1}}\left(x_{n}\right), \ldots, \alpha^{a_{n, k}}\left(x_{n}\right)\right)$ for some $f \in \mathscr{C}, k \geq 1$ and a sequence $\left(a_{i, j}\right)$ of integers. Set $f^{\prime}=\alpha \circ f \circ \alpha^{-1} \in \mathscr{C}$. Then $f=\alpha^{-1} \circ f^{\prime} \circ \alpha$ and we can calculate

$$
\begin{aligned}
t & =\alpha \circ s \\
& =\alpha \circ \alpha^{-1} \circ f^{\prime} \circ \alpha\left(\alpha^{a_{1,1}}\left(x_{1}\right), \ldots, \alpha^{a_{1, k}}\left(x_{1}\right), \ldots, \alpha^{a_{n, 1}}\left(x_{n}\right), \ldots, \alpha^{a_{n, k}}\left(x_{n}\right)\right) \\
& =f^{\prime}\left(\alpha^{a_{1,1}+1}\left(x_{1}\right), \ldots, \alpha^{a_{1, k}+1}\left(x_{1}\right), \ldots, \alpha^{a_{n, 1}+1}\left(x_{n}\right), \ldots, \alpha^{a_{n, k}+1}\left(x_{n}\right)\right) .
\end{aligned}
$$

To finish the induction, assume that $t=f\left(t_{1}, \ldots, t_{m}\right)$, where $f \in \mathscr{C}^{(m)}$ and $t_{l} \in$ $\langle\{\alpha\} \cup \mathscr{C}\rangle^{(n)}$ satisfies the induction hypothesis, $1 \leq l \leq m$. Then

$$
t_{l}=f_{l}\left(\alpha^{a_{1,1}^{l}}\left(x_{1}\right), \ldots, \alpha^{a_{1, k_{l}}^{l}}\left(x_{1}\right), \ldots, \alpha^{a_{n, 1}^{l}}\left(x_{n}\right), \ldots, \alpha^{a_{n, k_{l}}^{l}}\left(x_{n}\right)\right)
$$

for $f_{l} \in \mathscr{C}, k_{l} \geq 1$ and sequences $\left(a_{i, j}^{l}: 1 \leq i \leq n, 1 \leq j \leq k_{l}\right)$. By adding fictitious variables to the $f_{l}$, which we can do freely within a clone, we can assume that all $k_{l}$ are equal to some $k \geq 1$, and even that all sequences $\left(a_{i, j}^{l}: 1 \leq i \leq n, 1 \leq j \leq k_{l}\right)$ are identical with one sequence $\left(a_{i, j}: 1 \leq i \leq n, 1 \leq j \leq k\right)$. Then the $f_{l}$ are all of the same arity $k \cdot n$. Setting $g=f\left(f_{1}, \ldots, f_{m}\right) \in \mathscr{C}$, we obtain

$$
t=g\left(\alpha^{a_{1,1}}\left(x_{1}\right), \ldots, \alpha^{a_{1, k}}\left(x_{1}\right), \ldots, \alpha^{a_{n, 1}}\left(x_{n}\right), \ldots, \alpha^{a_{n, k}}\left(x_{n}\right)\right) .
$$

To make things more convenient, we consider permutations $\alpha$ which satisfy $\alpha^{2}=$ id; then the preceding lemma becomes

Lemma 11. If $\mathscr{C}$ is a symmetric clone and $\alpha \in \mathscr{S}$ satisfying $\alpha^{2}=\mathrm{id}$, and if $t \in \mathscr{O}^{(n)}$, then $t \in\langle\{\alpha\} \cup \mathscr{C}\rangle$ iff there exists $f \in \mathscr{C}^{(2 n)}$ such that

$$
t\left(x_{1}, \ldots, x_{n}\right)=f\left(\alpha\left(x_{1}\right), \ldots, \alpha\left(x_{n}\right), x_{1}, \ldots, x_{n}\right) .
$$

Proof. If $t$ has such a representation, then it obviously is an element $\langle\{\alpha\} \cup \mathscr{C}\rangle$. Conversely, let $t \in\langle\{\alpha\} \cup \mathscr{C}\rangle$. By the preceding lemma, $t$ has a representation

$$
t\left(x_{1}, \ldots, x_{n}\right)=f\left(\alpha^{a_{1,1}}\left(x_{1}\right), \ldots, \alpha^{a_{1, k}}\left(x_{1}\right), \ldots, \alpha^{a_{n, 1}}\left(x_{n}\right), \ldots, \alpha^{a_{n, k}}\left(x_{n}\right)\right)
$$

for some $k \geq 1$, a sequence $\left(a_{i, j}: 1 \leq i \leq n, 1 \leq j \leq k\right)$, and $f \in \mathscr{C}$. Now because $\alpha^{2}=\mathrm{id}$, all the exponents $a_{i, j}$ become either 0 or 1 . Since a clone is closed under identification of variables and under changing of the order of variables, as well as under addition of fictitious variables, we can assume that $\alpha^{1}\left(x_{i}\right)$ and $\alpha^{0}\left(x_{i}\right)$ occur exactly once in the representation for each $1 \leq i \leq n$, and that they occur in the desired order.

Definition 12. Let $n \geq 1$. We say that $\mathscr{H} \subseteq \mathscr{O}$ has the $n$-ary co-large approximation property iff for all $f \in \mathscr{O}^{(n)}$ and all co-large $S \subseteq X$ there exists $g \in \mathscr{H}^{(n)}$ such that $g\left\lceil_{S^{n}}=f\left\lceil_{S^{n}}\right.\right.$. We call the function $g$ an approximation to $f$ on $S$. $\mathscr{H}$ has the co-large approximation property iff it has the $n$-ary co-large approximation property for all $n \geq 1$.

A reformulation of the definition of the co-large approximation property which we will use heavily in the following is: $\mathscr{H} \subseteq \mathscr{O}$ has the $n$-ary co-large approximation property iff for all co-large $S \subseteq X$ and all functions $f: S^{n} \rightarrow X$ there is a function $g \in \mathscr{H}$ extending $f$ to $X^{n}$. We will give an example of a non-trivial clone having the 
co-large approximation property later in this paper (Proposition 25). The following is not surprising since all finitary operations are generated by binary operations.

Lemma 13. Let $\mathscr{C} \subseteq \mathscr{O}$ be a clone. Then $\mathscr{C}$ has the binary co-large approximation property iff it has the co-large approximation property.

Proof. It suffices to show that the binary co-large approximation property implies the co-large approximation property. Let $f \in \mathscr{O}^{(n)}$ and $S \subseteq X$ co-large. We want to find in $\mathscr{C}$ an approximation to $f$ on $S$. Without loss of generality, we can assume $S$ to be large. Fix a bijection $j: X \rightarrow S$ and set $h=j \circ f$. Then $h\left[X^{n}\right] \subseteq S$, hence $h \int_{S^{n}}$ is an operation on $S$. Because on every set the binary functions generate all finitary functions, $h\left\lceil_{S^{n}}\right.$ is generated by binary functions on $S$, all of which have extensions to $X$ in $\mathscr{C}$ by the binary co-large approximation property. Hence, there exists $g \in \mathscr{C}^{(n)}$ such that $g\left\lceil_{S^{n}}=h\left\lceil_{S^{n}}\right.\right.$. Moreover, again by the binary co-large approximation property of $\mathscr{C}$, there is $t \in \mathscr{C}^{(1)}$ such that $t \uparrow_{S}=j^{-1}$. Thus for $t \circ g \in \mathscr{C}$ we have $t \circ g \uparrow_{S^{n}}=j^{-1} \circ j \circ f\left\lceil_{S^{n}}=f \uparrow_{S^{n}}\right.$, so $t \circ g$ is an approximation to $f$ on $S$.

Lemma 14. Let $\mathscr{C}$ be symmetric and precomplete, and assume that $\mathscr{C} \nsupseteq \mathscr{S}$. Then $\mathscr{C}$ has the co-large approximation property.

Proof. Let $f \in \mathscr{O}^{(n)}$ and any co-large $S \subseteq X$ be given. Take $\alpha \in \mathscr{S}$ with support $X \backslash S$ and such that $\alpha^{2}=$ id, i.e., let $\alpha$ be the identity on $S$ and let its cycles on $X \backslash S$ be any partition of $X \backslash S$ into two-element sets. Because $\alpha$ has large support, Corollary 8 implies that $\alpha \notin \mathscr{C}$, and therefore $\langle\{\alpha\} \cup \mathscr{C}\rangle=\mathscr{O}$ as $\mathscr{C}$ is precomplete. By Lemma 111 there exists $g \in \mathscr{C}^{(2 n)}$ such that $f\left(x_{1}, \ldots, x_{n}\right)=$ $g\left(\alpha\left(x_{1}\right), \ldots, \alpha\left(x_{n}\right), x_{1}, \ldots, x_{n}\right)$. Set $h\left(x_{1}, \ldots, x_{n}\right)=g\left(x_{1}, \ldots, x_{n}, x_{1}, \ldots, x_{n}\right) \in \mathscr{C}$. Since $\alpha$ is the identity on $S$, we have $h\left(x_{1}, \ldots, x_{n}\right)=g\left(x_{1}, \ldots, x_{n}, x_{1}, \ldots, x_{n}\right)=$ $g\left(\alpha\left(x_{1}\right), \ldots, \alpha\left(x_{n}\right), x_{1}, \ldots, x_{n}\right)=f\left(x_{1}, \ldots, x_{n}\right)$ on $S$, whence $h$ is an approximation to $f$ on $S$.

Definition 15. We say that a function $f \in \mathscr{O}^{(1)}$ is generous iff all equivalence classes of its kernel are large. We set $\mathscr{I}_{0}$ to consist of all generous functions which are onto.

The unary clone $\mathscr{I}_{0}$ is an example of a symmetric proper submonoid of $\mathscr{O}^{(1)}$ having the unary co-large approximation property. Indeed, given any $f \in \mathscr{O}^{(1)}$ and any co-large $S \subseteq X$, then since $X \backslash S$ is large we can find $\gamma: X \backslash S \rightarrow X$ mapping $X \backslash S$ onto $X$ in such a way that $\gamma^{-1}[\{y\}]$ is large for all $y \in X$. Now set $g(x)=f(x)$ if $x \in S$, and $g(x)=\gamma(x)$ if $x \notin S$. Then $g^{-1}[\{y\}]$ is large for all $y \in X$ since $g$ extends $\gamma$, so $g \in \mathscr{I}_{0}$; moreover, $g$ is an approximation to $f$ on $S$ by its definition.

Let $f \in \mathscr{I}_{0}$, and fix any $x \in X$. Then we have the following possibilities:

- There exist $n \geq 0$ and $p \geq 1$ such that $f^{n}(x)=f^{n+p}(x)$.

- $x, f(x), f^{2}(x), \ldots$ are all distinct.

In the first case, choosing $p$ minimal with the property that there is $n \geq 0$ such that $f^{n}(x)=f^{n+p}(x)$, we call the set of all elements $y \in X$ such that there is $k \geq 0$ with $f^{k}(y)=x$ or $f^{k}(x)=y$, or equivalently the connectedness component of $x$ in the graph $(X, f)$, a $p$-snail. In the second case, we call the set of all elements of $X$ connected to $x$ a 0 -snail. This definition is independent of the element of a 
snail we are looking at, that is, it depends only on the connectedness component of $x$ in $(X, f)$. Indeed, let $y \in X$ be connected to $x$; then there exists $k \geq 0$ such that either $f^{k}(y)=x$ or $f^{k}(x)=y$. Assume without loss of generality the first case holds. Now if $f^{n}(x)=f^{n+p}(x)$ for some $n \geq 0$ and $p \geq 1$, and if $p$ is minimal with this property, then $f^{k+n}(y)=f^{k+n+p}(y)$, so in particular $y$ does not think it is part of an infinite snail. Suppose there exist $j \geq 0$ and $1 \leq q<p$ such that $f^{j}(y)=f^{j+q}(y)$. Then $f^{j+k}(y)=f^{j+q+k}(y)$ and so $f^{j}(x)=f^{j+q}(x)$, contradicting the minimality of $p$. Hence, $y$ thinks it is in a $p$-snail too. If on the other hand $x, f(x), f^{2}(x), \ldots$ are all distinct then the same holds for $y$, for if $f^{j}(y)=f^{j+q}(y)$ for $j \geq 0$ and $q \geq 1$, then $f^{j}(x)=f^{j+k}(y)=f^{j+q+k}(y)=f^{j+q}(x)$, contradiction.

Definition 16. We call a function $f \in \mathscr{I}_{0}$ rich iff it has a large number of $p$-snails for all $p \geq 0$.

If $\left(a_{p}\right)_{p \in \omega}$ is any sequence of cardinals $\leq \kappa$ which is not constantly zero, then there exists a function $f \in \mathscr{I}_{0}$ whose number of $p$-snails is $a_{p}$, for all $p \in \omega$. Indeed, let $p>0$, take $x_{0}, \ldots, x_{p-1} \in X$, and define $f\left(x_{i}\right)=x_{i+1}$ for $0 \leq i \leq p-2$, and $f\left(x_{p-1}\right)=x_{0}$. Moreover, let $f$ map $X \backslash\left\{x_{0}, \ldots, x_{p-1}\right\}$ onto $X$ in such a way that the preimage of every $y \in X$ is large. Now set $X^{\prime}$ to consist of all $x \in X$ such that there is $k \geq 0$ with $f^{k}(x) \in\left\{x_{0}, \ldots, x_{p-1}\right\}$. Then the restriction of $f$ to $X^{\prime}$ is a function on $X^{\prime}$ which is onto and generous, and which has exactly one $p$-snail and no other snails. Since $\left|X^{\prime}\right|=|X|$, such a function exists also on $X$. Similarly we can prove the existence of functions having only one 0 -snail and no other snails. By taking the union over functions on disjoint sets, all of which have only one snail, according to the sequence $\left(a_{p}\right)_{p \in \omega}$, one obtains the function whose number of $p$-snails is $a_{p}$, for all $p \in \omega$. In particular, rich functions exist.

Lemma 17. Let $\mathscr{H} \subseteq \mathscr{O}$ have the unary co-large approximation property. Then there exists a rich $f \in \mathscr{H}^{(1)}$.

Proof. Fix any large and co-large $S \subseteq X$, and some $T \subseteq S$ such that $T$ and $S \backslash T$ are large. Take any $m_{1}: T \rightarrow T$ which is rich (as an operation on the base set $T)$. Now let $m_{2}: S \backslash T \rightarrow X$ be so that $m_{2}^{-1}[\{y\}]$ is large for every $y \in X$. Set $m=m_{1} \cup m_{2}: S \rightarrow X$; by the unary co-large approximation property of $\mathscr{H}$, there is a function $f \in \mathscr{H}^{(1)}$ with $f \uparrow_{S}=m$. Because $f$ extends $m_{2}$, every $y \in X$ has a large preimage under $f$; thus, $f \in \mathscr{I}_{0}$. Being identical with $m_{1}$ on $T, f$ has a large number of $p$-snails for all $p \geq 0$, and we see that $f$ is rich.

Lemma 18. Let $f, g \in \mathscr{I}_{0}$. Then $f$ and $g$ have the same number of $p$-snails for all $p \geq 0$ iff there exists $\gamma \in \mathscr{S}$ such that $f=\gamma^{-1} \circ g \circ \gamma$.

Proof. If $f=\gamma^{-1} \circ g \circ \gamma$, then the structures $(X, f)$ and $(X, g)$ are isomorphic via $\gamma$; this obviously implies that if $x \in X$ is part of a $p$-snail of $f$, then $\gamma(x)$ is part of a $p$-snail of $g$. In particular, $f$ and $g$ have the same number of $p$-snails for all $p \geq 0$. Let on the other hand $f$ and $g$ have the same number of $p$-snails for all $p \geq 0$; we will construct an isomorphism $\gamma:(X, f) \rightarrow(X, g)$. Assume first that $f$ and $g$ have exactly one $p$-snail and no other snails, where $p>0$. There exist $a, b \in X$ such that $f^{p}(a)=a$ and $g^{p}(b)=b$. Set $A=\left\{a, f(a), \ldots, f^{p-1}(a)\right\}$ and $B=$ $\left\{b, g(b), \ldots, g^{p-1}(b)\right\}$; then, since we are in a $p$-snail, $|A|=|B|=p$. Now for every $x \in X$ there is a minimal $n \geq 0$ such that $f^{n}(x) \in A$; we say that $x$ is on the $n$-th level with respect to $f$. Levels with respect to $g$ are defined analogously. We define $\gamma$ by induction over levels. Set $\gamma\left(f^{k}(a)\right)=g^{k}(b)$, for all $0 \leq k \leq p-1$; that 
defines $\gamma$ on $A$, which constitutes exactly the 0 -th level of $f$. To define $\gamma$ on the first level, consider the sets $f^{-1}[\{x\}] \backslash A$ and $g^{-1}[\{\gamma(x)\}] \backslash B$, for all $x \in A$. Since they are both large, we can map the first bijectively onto the latter; we extend $\gamma$ by such a bijection. This defines $\gamma$ for level 1. Say $\gamma$ has already been defined for all $x$ of a level smaller than $n$, where $n \geq 2$. Let $x$ be at level $n-1$ and take again the sets $f^{-1}[\{x\}]$ and $g^{-1}[\{\gamma(x)\}]$; this time we do not have to remove the elements of $A$ and $B$, since they are not mapped to level $n-1$, as $n \geq 2$. The preimages are both large, so as on the first level, we map the first bijectively onto the latter, and extend $\gamma$ by such a bijection. This defines $\gamma$ for level $n$, since every $y$ on level $n$ is an element of the preimage of exactly one $x$ of level $n-1$. Moreover, because every $x \in X$ appears on exactly one level, $\gamma$ is a function on $X$, and it maps the $n$-th level with respect to $f$ to the $n$-th level with respect to $g$, for all $n \geq 0$. We claim that $\gamma$ is injective. Indeed, to see this we prove by induction over levels that $\gamma$ is injective on each level, which is sufficient as it maps distinct levels of $f$ to distinct levels of $g$. It is clear that $\gamma$ is injective on $A$, by definition. So let $x, y$ be distinct elements of the $n$-th level, where $n \geq 1$. If $f(x) \neq f(y)$, then by induction hypothesis $\gamma(f(x)) \neq \gamma(f(y))$ and so $\gamma(x) \neq \gamma(y)$ since $\gamma(x) \in g^{-1}[\{\gamma(f(x))\}]$ but $\gamma(y) \in g^{-1}[\{\gamma(f(y))\}]$. If $f(x)=f(y)$, then, since both $x$ and $y$ are in $f^{-1}[\{f(x)\}] \backslash A$, and since $\gamma$ maps $f^{-1}[\{f(x)\}] \backslash A$ bijectively onto $g^{-1}[\{\gamma(f(x))\}] \backslash B$, we again have that $\gamma(x) \neq \gamma(y)$, and the induction is complete. To prove that $\gamma$ is surjective, we again proceed by induction. If $y \in B$, then it is in the range of $\gamma$. Now let $y$ be on level $n$ with respect to $g$, where $n \geq 1$, and assume that all elements of lower level are in the range of $\gamma$; then $g(y) \in \gamma[X]$ by induction hypothesis. Let $z$ be the element for which $\gamma(z)=g(y)$. By definition of $g, f^{-1}[\{z\}] \backslash A$ is mapped onto $g^{-1}[\{\gamma(z)\}] \backslash B=g^{-1}[\{g(y)\}] \backslash B$; therefore, $y$ has an element in $f^{-1}[\{z\}]$ mapped to it, so $g$ is surjective. Now $\gamma(f(x))=g(\gamma(x))$ for all $x \in X$ by construction of $\gamma$, so $\gamma$ is an isomorphism.

Assume next that $f$ and $g$ have only one 0 -snail, and no other snails. Take any $a, b \in X$, and set $A=\left\{a, f(a), f^{2}(a), \ldots\right\}$ and $B=\left\{b, g(b), g^{2}(b), \ldots\right\}$. Define $\gamma\left(f^{k}(a)\right)=g^{k}(b)$, for all $k \geq 0$; then proceed again defining $\gamma$ by induction over levels. As before, one checks that $\gamma$ is an isomorphism.

Now if $f$ and $g$ have the same number of $p$-snails for all $p \geq 0$, then fix for every $p \geq 0$ a bijection $\sigma_{p}$ from the $p$-snails of $f$ onto the $p$-snails of $g$. By the preceding discussion, if $\partial$ is any $p$-snail of $f$, we can construct an isomorphism $\gamma_{\partial}$ from $(\partial, f)$ onto $\left(\sigma_{p}(\partial), g\right)$. If we set $\gamma$ to be the union over all $\gamma_{\partial}$, for all snails $\partial$ of $f$, we obtain an isomorphism between $(X, f)$ and $(X, g)$.

Lemma 19. If $\mathscr{C}$ is a symmetric clone with the unary co-large approximation property, then $\mathscr{C}$ contains all rich functions.

Proof. By Lemma $17 \mathscr{C}$ contains a rich function. Hence it contains all rich functions by the preceding lemma.

Recall from Theorem 2 that $\mathscr{E} \subseteq \mathscr{O}^{(1)}$ consists of those functions in $\mathscr{O}^{(1)}$ which take all but a small set of values.

Lemma 20. If $\mathscr{C}$ is a symmetric clone which has the unary co-large approximation property, and if $\mathscr{C}^{(1)} \subseteq \mathscr{E}$, then $\mathscr{C} \subseteq \operatorname{Pol}(\mathscr{E})$.

Proof. Assuming that there exists $f \in \mathscr{C} \backslash \operatorname{Pol}(\mathscr{E})$ we find $g \in \mathscr{C}^{(1)}$ such that $g \notin \mathscr{E}$. Because $f \notin \operatorname{Pol}(\mathscr{E})$, there exist $\alpha_{1}, \ldots, \alpha_{n} \in \mathscr{E}$ such that $f\left(\alpha_{1}, \ldots, \alpha_{n}\right) \notin \mathscr{E}$. Fix 
$A \subseteq X$ small such that $A \cup \alpha_{i}[X]=X$ for all $1 \leq i \leq n$; this is possible since the range of all $\alpha_{i}$ is co-small. Choose $S \subseteq X \backslash A$ large and co-large such that $f\left(\alpha_{1}, \ldots, \alpha_{n}\right)[X] \cup\{f(x, \ldots, x): x \in S\}$ is still co-large. Set $Y=X \backslash(A \cup S)$. Then $Y$ is large, implying that we can find $\gamma \in \mathscr{O}^{(1)}$ mapping $Y$ onto $X$. Now define for all $1 \leq i \leq n$

$$
\beta_{i}(x)= \begin{cases}\alpha_{i} \circ \gamma(x), & x \in Y \\ x, & x \in A \cup S .\end{cases}
$$

Then $f\left(\beta_{1}, \ldots, \beta_{n}\right)[X]=f\left(\beta_{1}, \ldots, \beta_{n}\right)[Y] \cup f\left(\beta_{1}, \ldots, \beta_{n}\right)[A \cup S]=f\left(\alpha_{1}, \ldots, \alpha_{n}\right)[X] \cup$ $\{f(x, \ldots, x): x \in A \cup S\}$ is co-large. Let $h \in \mathscr{O}^{(1)}$ be so that $h[S]=S, h\left\lceil_{S}\right.$ is a rich function on the large set $S$, and such that it maps $X \backslash S$ onto $X$ in such a way that every $x \in X$ has a large preimage in $X \backslash S$ under $h$. Then already its definition on $X \backslash S$ guarantees that $h$ is onto and that all classes of its kernel are large, so $h \in \mathscr{I}_{0}$. Because $h$ is onto we have that for all $1 \leq i \leq n$, $\beta_{i} \circ h[X]=\beta_{i}[X]=\beta_{i}[Y] \cup A \cup S=\alpha_{i}[X] \cup A \cup S=X$. Also, since all classes in the kernel of $h$ are large, so are those of $\beta_{i} \circ h$; therefore, $\beta_{i} \circ h \in \mathscr{I}_{0}$. Moreover, $\beta_{i} \circ h$ has a large number of $p$-snails for all $p \geq 0$, since already $h\left\lceil_{S}\right.$ has this property and $\beta_{i} \circ h\left\lceil_{S}=h\left\lceil_{S}\right.\right.$ as $\beta_{i} \uparrow_{S}=\mathrm{id}_{S}$. Hence, $\beta_{i} \circ h$ is rich and therefore an element of $\mathscr{C}$ by Lemma 19] But $f\left(\beta_{1} \circ h, \ldots, \beta_{n} \circ h\right)[X]=f\left(\beta_{1}, \ldots, \beta_{n}\right)[X]$ is co-large so that it suffices to set $g=f\left(\beta_{1} \circ h, \ldots, \beta_{n} \circ h\right) \in \mathscr{C}$.

Remember that the monoid $\mathscr{F} \subseteq \mathscr{O}^{(1)}$ is the union of $\mathscr{E}$ and all constant functions.

Lemma 21. If $\mathscr{C}$ is a symmetric clone which has the unary co-large approximation property, and if $\mathscr{C}^{(1)} \nsubseteq \mathscr{E}$ and $\mathscr{C}^{(1)} \subseteq \mathscr{F}$, then $\mathscr{C} \subseteq \operatorname{Pol}(\mathscr{F})$.

Proof. A slight modification of the proof of the preceding lemma yields that assuming there is $f \in \mathscr{C} \backslash \operatorname{Pol}(\mathscr{F})$, we can find $g \in \mathscr{C}^{(1)}$ such that $g \notin \mathscr{F}$. So let $f \in \mathscr{C} \backslash \operatorname{Pol}(\mathscr{F})$; then there exist $\alpha_{1}, \ldots, \alpha_{n} \in \mathscr{F}$ such that $f\left(\alpha_{1}, \ldots, \alpha_{n}\right) \notin \mathscr{F}$. Now observe that the conditions $\mathscr{C}^{(1)} \nsubseteq \mathscr{E}$ and $\mathscr{C}^{(1)} \subseteq \mathscr{F}$ imply that $\mathscr{C}^{(1)}$ contains a constant function; hence it contains all constant functions as it is symmetric. The functions $\alpha_{1}, \ldots, \alpha_{n} \in \mathscr{F}$ are either constant or almost surjective; assume $\alpha_{1}, \ldots, \alpha_{k}$ are constant, and $\alpha_{k+1}, \ldots, \alpha_{n}$ almost surjective, where $1 \leq k<n$. Note that $k=n$ is impossible for otherwise $f\left(\alpha_{1}, \ldots, \alpha_{n}\right)$ would be constant and thereby an element of $\mathscr{F}$. Consider $f\left(\alpha_{1}, \ldots, \alpha_{k}, x_{k+1}, \ldots, x_{n}\right)$ as a $(n-k)$-ary function $\tilde{f}$ of the variables $x_{k+1}, \ldots, x_{n}$. By the proof of the preceding lemma, we can find rich functions $\beta_{i} \circ h$ for $k+1 \leq i \leq n$ such that $g=\tilde{f}\left(\beta_{k+1} \circ h, \ldots, \beta_{n} \circ h\right)$ has co-large range. It also follows from that proof that $g[X] \supseteq \tilde{f}\left(\alpha_{k+1}, \ldots, \alpha_{n}\right)[X]=f\left(\alpha_{1}, \ldots, \alpha_{n}\right)[X]$, and so $g$ is not constant. Therefore $g \notin \mathscr{F}$. Now it is enough to observe that $g=f\left(\alpha_{1}, \ldots, \alpha_{k}, \beta_{k+1} \circ h, \ldots, \beta_{n} \circ h\right)$, and that all functions which appear here as arguments of $f$ are either constant or rich and thus elements of $\mathscr{C}$, by Lemma 19] Whence, $g \in \mathscr{C}^{(1)}$, contradicting the assumption $\mathscr{C}^{(1)} \subseteq \mathscr{F}$.

Lemma 22. If $\mathscr{C}$ is a symmetric clone which has the unary co-large approximation property, and if $\mathscr{C}^{(1)} \nsubseteq \mathscr{F}$, then $\mathscr{X}=\left\{\rho \in \mathscr{O}^{(1)}:|\rho[X]| \leq 2\right.$ and $\rho$ is generous $\}$ is contained in $\mathscr{C}$.

Proof. To start with, let $\rho \in \mathscr{X}$ be so that it takes two distinct values $c_{1}, c_{2} \in X$, and write $\theta_{i}=\rho^{-1}\left[\left\{c_{i}\right\}\right], i=1,2$. Take any $f \in \mathscr{C}^{(1)} \backslash \mathscr{F}$. Since $f$ is not constant there exist $a_{1} \neq a_{2}$ in the range of $f$. Define $s: f[X] \rightarrow\left\{a_{1}, a_{2}\right\}$ by $s\left(a_{1}\right)=a_{1}$ 
and $s(x)=a_{2}$ for all $x \neq a_{1}$. Since $f \notin \mathscr{F}$ we have that $f[X]$ is co-large, and so the unary co-large approximation property of $\mathscr{C}$ implies that we can extend $s$ to a function $g \in \mathscr{C}^{(1)}$. Choose any rich function $h \in \mathscr{O}^{(1)}$; then $h \in \mathscr{C}$ by Lemma 19] Therefore, $g \circ f \circ h$ is an element of $\mathscr{C}$ as well. Now since $h$ is generous, so is $g \circ f \circ h$. Also, since $h$ is onto and by the construction of $g, g \circ f \circ h[X]=g \circ f[X]=\left\{a_{1}, a_{2}\right\}$. Write $\zeta_{i}=(g \circ f \circ h)^{-1}\left[\left\{a_{i}\right\}\right], i=1,2$. Since the $\zeta_{i}$ and the $\theta_{i}$ are large, there exists $\gamma \in \mathscr{S}$ mapping $\theta_{i}$ onto $\zeta_{i}$, for $i=1,2$. Then $\gamma^{-1} \circ g \circ f \circ h \circ \gamma$ is in $\mathscr{C}$ as $\mathscr{C}$ is symmetric, and maps $\theta_{i}$ to $\gamma^{-1}\left(a_{i}\right), i=1,2$. Now let $t \in \mathscr{C}^{(1)}$ be so that it maps $\gamma^{-1}\left(a_{i}\right)$ to $c_{i}, i=1,2$. We can find such a $t$ in $\mathscr{C}$ by the unary co-large approximation property. Then $t \circ \gamma^{-1} \circ g \circ f \circ h \circ \gamma \in \mathscr{C}$, and it maps $\theta_{i}$ to $c_{i}$, $i=1,2$. Whence, it equals $\rho$ so that we infer $\rho \in \mathscr{C}$. Therefore $\mathscr{C}$ contains all functions in $\mathscr{X}$ which take two values.

Now to see that $\mathscr{C}$ contains the constant functions as well, let $c \in X$, and let $f$ be as before. By the unary co-large approximation property, we can find $q \in \mathscr{C}^{(1)}$ mapping all elements of the co-large range of $f$ to $c$. Then $q \circ f \in \mathscr{C}$ is constant with value $c$ and we are done.

Lemma 23. If $\mathscr{C}$ is a symmetric clone which has the co-large approximation property, and if $\mathscr{C} \supseteq \mathscr{X}$, then there exists $g \in \mathscr{C}^{(1)}$ having large and co-large range.

Proof. Choose any large and co-large $T \subseteq X$, and any element $0 \in T$. Fix $f \in \mathscr{C}^{(2)}$ such that $f[\{0\} \times X]$ and $f[X \times\{0\}]$ are large, and such that $f \uparrow_{T^{2}}$ is constantly 0 . To obtain $f$, let $S \subseteq X \backslash T$ be large and so that $T \cup S$ is co-large, and let $\gamma: S \rightarrow X$ be onto. Then define a partial binary operation $m$ to yield constantly 0 on $T^{2}$, and to satisfy $m(s, 0)=m(0, s)=\gamma(s)$ for all $s \in S$. Since the domain of $m$ is contained in $(S \cup T)^{2}$ and $S \cup T$ is co-large, by the co-large approximation property we can extend $m$ to $f \in \mathscr{C}^{(2)}$. Clearly, $f$ yields constantly 0 on $T^{2}$ as $m$ does, and $f[\{0\} \times X] \supseteq f[\{0\} \times S]=m[\{0\} \times S]=\gamma[S]=X$, and the same holds for $f[X \times\{0\}]$, so $f$ is indeed as desired. We distinguish two cases.

Case 1. For all $c \in X$ it is true that $f[X \times\{c\}]$ and $f[\{c\} \times X]$ are co-small. Then consider an arbitrary large and co-large $A \subseteq X$ with $0 \notin A$. Set $\Gamma=$ $f^{-1}[X \backslash A] \subseteq X^{2}$ and let $\alpha: X \backslash T \rightarrow \Gamma$ be onto. By the assumption for this case, $f[X \times\{c\}] \backslash A$ and $f[\{c\} \times X] \backslash A$ are still large for all $c \in X$. Thus the components $\alpha_{i}=\pi_{i}^{2} \circ \alpha$ hit every $c \in X$ at a large number of arguments, $i=1,2$. Moreover, by taking the union with any rich function on the base set $T$, we can extend the $\alpha_{i}$ to $T$ so that $\alpha_{i}[T]=T$ and $\alpha_{i} \Gamma_{T}$ is rich. The resulting operations $\alpha_{i} \in \mathscr{O}^{(1)}$ still hit every $c \in X$ at a large number of arguments, so they are elements of $\mathscr{I}_{0}$. Also, since they already have a large number of $p$-snails on $T$ for all $p \geq 0$, they certainly still have this property on $X$. Therefore they are rich and hence elements of $\mathscr{C}^{(1)}$ by Lemma 19] But now setting $g=f\left(\alpha_{1}, \alpha_{2}\right) \in \mathscr{C}$ we have that $g[X]=g[X \backslash T] \cup g[T]=f\left(\alpha_{1}, \alpha_{2}\right)[X \backslash T] \cup\{0\}=f\left[X^{2}\right] \backslash A$ is large and co-large and we are done.

Case 2. There exists $c \in X$ such that either $f[X \times\{c\}]$ or $f[\{c\} \times X]$ is co-large, say without loss of generality this is the case for $f[\{c\} \times X]$. Since $f[\{0\} \times X]$ is large we can choose $\Gamma \subseteq X$ large and co-large such that $f[\{0\} \times \Gamma]$ is large and such that $f[\{c\} \times X] \cup f[\{0\} \times \Gamma]$ is still co-large. Take moreover a rich $\beta \in \mathscr{I}_{0}$; then $\beta \in \mathscr{C}$ by Lemma 19 Now we define $\alpha \in \mathscr{O}^{(1)}$ by

$$
\alpha(x)= \begin{cases}0, & \beta(x) \in \Gamma \\ c, & \text { otherwise. }\end{cases}
$$


The range of $\alpha$ equals $\{0, c\}$, and the preimage of both values under $\alpha$ is large. Hence $\alpha \in \mathscr{X} \subseteq \mathscr{C}$. Thus it is enough to set $g=f(\alpha, \beta) \in \mathscr{C}$ and observe that $g[X]=f[\{c\} \times(X \backslash \Gamma)] \cup f[\{0\} \times \Gamma]$ is large and co-large.

Lemma 24. Assume that $\mathscr{C}$ is a symmetric clone with the co-large approximation property. If there is $g \in \mathscr{C}^{(1)}$ with large and co-large range, then $\mathscr{C}=\mathscr{O}$.

Proof. Because $\mathscr{C}$ has the co-large approximation property, by Lemma 17 there exists a rich $h \in \mathscr{C}^{(1)}$. The function $g^{\prime}=g \circ h \in \mathscr{C}^{(1)}$ is generous and has large and co-large range. Set $S=g^{\prime}[X] \subseteq X$. There exists $\gamma \in \mathscr{S}$ with the property that for all distinct $x, y \in X$ it is true that $g^{\prime}(x)=g^{\prime}(y)$ implies $g^{\prime} \circ \gamma(x) \neq g^{\prime} \circ \gamma(y)$. Indeed, let $\left\{\theta_{i}\right\}_{i \in \kappa}$ be an enumeration of the classes of the kernel of $g^{\prime}$, and enumerate the elements of those classes by $\theta_{i}=\left\{x_{i}^{j}\right\}_{j \in \kappa}$, for all $i \in \kappa$. Now define $\gamma$ by $\gamma\left(x_{i}^{j}\right)=x_{j}^{i}$ for all $i, j \in \kappa$. Every $x \in X$ is equal to some $x_{j}^{i}$, and therefore has the element $x_{i}^{j}$ mapped to it by $\gamma$; hence $\gamma$ is surjective. If $x_{i}^{j} \neq x_{p}^{q}$, then $i \neq p$ or $j \neq q$ and thus $\gamma\left(x_{i}^{j}\right)=x_{j}^{i} \neq x_{q}^{p}=\gamma\left(x_{p}^{q}\right)$, and we see that $\gamma$ is injective. Now if $g^{\prime}(x)=g^{\prime}(y)$ for $x \neq y$, then $x, y \in \theta_{i}$ for some $i \in \kappa$, and so there are distinct $j, k \in \kappa$ such that $x=x_{i}^{j}$ and $y=x_{i}^{k}$. But then $g^{\prime}(\gamma(x))=g^{\prime}\left(x_{j}^{i}\right)$ and $g^{\prime}(\gamma(y))=g^{\prime}\left(x_{k}^{i}\right)$ are not equal as $x_{j}^{i}$ and $x_{k}^{i}$ belong to different kernel classes of $g^{\prime}$. Thus $\gamma$ has the desired properties. Set $g^{\prime \prime}=\gamma^{-1} \circ g^{\prime} \circ \gamma$; then $g^{\prime \prime} \in \mathscr{C}$ because $\mathscr{C}$ is symmetric, and $g^{\prime \prime}$ still satisfies $g^{\prime \prime}(x) \neq g^{\prime \prime}(y)$ whenever $g^{\prime}(x)=g^{\prime}(y)$ and $x, y \in X$ are distinct. Since the range of $g^{\prime \prime}$ is large and co-large, the co-large approximation property guarantees that we can find $f \in \mathscr{C}^{(1)}$ which maps $g^{\prime \prime}[X]$ injectively onto $S$. Set $g^{\prime \prime \prime}=f \circ g^{\prime \prime}$. Then the function $t(x)=\left(g^{\prime}(x), g^{\prime \prime \prime}(x)\right)$ maps $X$ injectively into $S^{2}$. For both $g^{\prime}$ and $g^{\prime \prime \prime}$ take only values in $S$, and if $g^{\prime}(x)=g^{\prime}(y)$ for distinct $x, y \in X$, then $g^{\prime \prime \prime}(x) \neq g^{\prime \prime \prime}(y)$. Therefore, the function $s(x, y)=\left(g^{\prime}(x), g^{\prime \prime \prime}(x), g^{\prime}(y), g^{\prime \prime \prime}(y)\right)$ maps $X^{2}$ injectively into $S^{4}$. Now let an arbitrary $q \in \mathscr{O}^{(2)}$ be given. We can find $m \in \mathscr{C}^{(4)}$ satisfying

$$
m\left(g^{\prime}(x), g^{\prime \prime \prime}(x), g^{\prime}(y), g^{\prime \prime \prime}(y)\right)=q(x, y)
$$

for all $x, y \in X$. This is because distinct pairs in $X^{2}$ yield distinct quadruples in $S^{4}$ via $s$, and we can define $m$ on $S^{4}$ as required by $q$ and extend it to a function in $\mathscr{C}^{(4)}$ by the co-large approximation property. The equality implies $q \in \mathscr{C}$ and so $\mathscr{C} \supseteq \mathscr{O}^{(2)}$ since $q$ was arbitrary. Whence, $\mathscr{C}=\mathscr{O}$.

Proposition 25. $\operatorname{Pol}(\mathscr{E})$ and $\operatorname{Pol}(\mathscr{F})$ have the co-large approximation property.

Proof. Let $f \in \mathscr{O}^{(2)}$ and $S \subseteq X$ be co-large. We construct an approximation $g$ to $f$ on $S$ which is an element of both $\operatorname{Pol}(\mathscr{E})$ and $\operatorname{Pol}(\mathscr{F})$, proving that these clones have the binary co-large approximation property and hence the co-large approximation property by Lemma 13 Let $\gamma \in \mathscr{O}^{(1)}$ be so that it maps $X \backslash S$ onto $X$. Define $g \in \mathscr{O}^{(2)}$ by

$$
g\left(x_{1}, x_{2}\right)= \begin{cases}f\left(x_{1}, x_{2}\right), & x_{1}, x_{2} \in S \\ \gamma\left(x_{1}\right), & x_{1} \notin S \\ \gamma\left(x_{2}\right), & x_{1} \in S \wedge x_{2} \notin S .\end{cases}
$$

Then $g$ and $f$ agree on $S^{2}$ so that $g$ is an approximation to $f$ on $S$. To see that $g \in \operatorname{Pol}(\mathscr{E})$, let $g_{1}, g_{2} \in \mathscr{E}$ be given. Since the range of $g_{1}$ is co-small, we have that $g_{1}$ 
misses only a small number of elements of $X \backslash S$, and therefore $\gamma\left[(X \backslash S) \cap g_{1}[X]\right]$ is cosmall. Now $g\left(g_{1}, g_{2}\right)(x)=\gamma\left(g_{1}(x)\right)$ whenever $g_{1}(x) \in X \backslash S$. Hence, $g\left(g_{1}, g_{2}\right)[X] \supseteq$ $\gamma\left[(X \backslash S) \cap g_{1}[X]\right]$ is co-small, so that $g\left(g_{1}, g_{2}\right) \in \mathscr{E}$. Whence, $g \in \operatorname{Pol}(\mathscr{E})$ and $\operatorname{Pol}(\mathscr{E})$ has the co-large approximation property.

We now show that $g \in \operatorname{Pol}(\mathscr{F})$. Let $g_{1}, g_{2} \in \mathscr{F}$, i.e. they are either constant or almost surjective. If both $g_{1}$ and $g_{2}$ are almost surjective, then so is $g\left(g_{1}, g_{2}\right)$ as we have just seen, so $g\left(g_{1}, g_{2}\right) \in \mathscr{F}$. If on the other hand both functions are constant, then the composite $g\left(g_{1}, g_{2}\right)$ is constant as well so that again $g\left(g_{1}, g_{2}\right) \in \mathscr{F}$. Next assume that $g_{2}$ is constant and $g_{1}$ is almost surjective. Then exactly the argument of the preceding paragraph yields that $g\left(g_{1}, g_{2}\right)$ is almost surjective, since in that argument we needed only that $g_{1}$ is almost surjective, and no assumptions on $g_{2}$. Therefore we obtain $g\left(g_{1}, g_{2}\right) \in \mathscr{F}$ also in this case. Finally, consider the case where $g_{1}$ is constant and $g_{2}$ is almost surjective. We distinguish two subcases: If $g_{1}$ constantly yields a value $c$ in $X \backslash S$, then $g\left(g_{1}, g_{2}\right)(x)=\gamma(c)$ for all $x \in X$, so $g\left(g_{1}, g_{2}\right)$ is constant. If on the other hand $g_{1}$ is constant with value $c \in S$, then by definition of $g$ we have that $g\left(g_{1}, g_{2}\right)(x)=\gamma\left(g_{2}(x)\right)$ whenever $g_{2}(x) \in X \backslash S$. Since $g_{2}$ is almost surjective, it misses only a small number of elements in $X \backslash S$, and so $\gamma\left[(X \backslash S) \cap g_{2}[X]\right]$ is co-small. Hence, since $g\left(g_{1}, g_{2}\right)[X] \supseteq \gamma\left[(X \backslash S) \cap g_{2}[X]\right]$, we see that $g\left(g_{1}, g_{2}\right)$ is almost surjective. In either case, $g\left(g_{1}, g_{2}\right) \in \mathscr{F}$ and thus $g \in \operatorname{Pol}(\mathscr{F})$. Therefore $\operatorname{Pol}(\mathscr{F})$ has the co-large approximation property.

Proposition 26. The only symmetric precomplete clones having the co-large approximation property are $\operatorname{Pol}(\mathscr{E})$ and $\operatorname{Pol}(\mathscr{F})$.

Proof. We know from Theorem 2 that $\operatorname{Pol}(\mathscr{E})$ and $\operatorname{Pol}(\mathscr{F})$ are precomplete and symmetric; by the preceding proposition, both clones have the co-large approximation property. Suppose $\mathscr{C}$ is a symmetric clone having the co-large approximation property, and which is distinct from those two clones. If $\mathscr{C}^{(1)} \subseteq \mathscr{E}$, then $\mathscr{C} \subseteq \operatorname{Pol}(\mathscr{E})$ by Lemma $\left[20\right.$ and so $\mathscr{C}$ is not precomplete. Moreover, if $\mathscr{C}^{(1)} \subseteq \mathscr{F}$ and $\mathscr{C} \nsubseteq \mathscr{E}$, then $\mathscr{C} \subseteq \operatorname{Pol}(\mathscr{F})$ by Lemma 21] hence $\mathscr{C}$ is not precomplete either. Finally, if $\mathscr{C}^{(1)} \nsubseteq \mathscr{F}$, then $\mathscr{C} \supseteq \mathscr{X}$ by Lemma 22 and thus $\mathscr{C}$ contains a unary function with large and co-large range by Lemma 23 Hence, $\mathscr{C}=\mathscr{O}$ by Lemma 24

Proof of Theorem 3 . Assume there exists a symmetric and precomplete clone $\mathscr{C}$ with $\mathscr{C} \nsupseteq \mathscr{S}$. Then $\mathscr{C}$ has the co-large approximation property by Lemma 14] so $\mathscr{C}$ has to equal either $\operatorname{Pol}(\mathscr{E})$ or $\operatorname{Pol}(\mathscr{F})$ by the preceding proposition. Contradiction.

\section{REFERENCES}

[1] R. Baer. Die Kompositionsreihe der Gruppe aller eineindeutigen Abbildungen einer unendlichen Menge auf sich. Stud. Math., 5:15-17, 1934.

[2] G. P. Gavrilov. On functional completeness in countable-valued logic. Problemy Kibernetiki, 15:5-64, 1965. In Russian.

[3] M. Goldstern and S. Shelah. Clones from creatures. Transactions of the American Mathematical Society. to appear.

[4] M. Goldstern and S. Shelah. Large intervals in the clone lattice. preprint [GoSh:747].

[5] M. Goldstern and S. Shelah. Clones on regular cardinals. Fundam. Math., 173(1):1-20, 2002.

[6] L. Heindorf. The maximal clones on countable sets that include all permutations. Algebra univers., 48:209-222, 2002.

[7] Nguen Van Khoa. On the structure of self-dual closed classes of three-valued logic $P_{3}$. Diskretn. Mat., 4(4):82-95, 1992. In Russian. 
[8] Nguen Van Khoa. Families of closed classes that are preserved by all automorphisms. Diskretn. Mat., 5(4):87-108, 1993. In Russian.

[9] Nguen Van Khoa. Description of closed classes that are preserved by all inner automorphisms of $k$-valued logic. Dokl. Akad. Nauk Belarusi, 38(3):16-19, 1994. In Russian.

[10] I. Levi and R. B. McFadden. $S_{n^{-}}$normal semigroups. Proc. Edinb. Math. Soc., 37:471-476, 1994.

[11] S. S. Marchenkov. Basic relations for the $S$-classification of functions of multivalued logic. Diskretn. Mat., 8(1):99-128, 1996. In Russian.

[12] S. S. Marchenkov. G-precomplete classes of many-valued logic. Diskretn. Anal. Issled. Oper., 3(3):47-70, 1996. In Russian.

[13] M. Pinsker. Maximal clones on uncountable sets that include all permutations. Algebra univers. to appear.

[14] M. Pinsker. The number of unary clones containing the permutations on an infinite set. Acta Sci. Math. to appear.

[15] I. G. Rosenberg. The set of maximal closed classes of operations on an infinite set $A$ has cardinality $2^{2^{|A|}}$. Arch. Math. (Basel), 27:561-568, 1976.

[16] J. Schreier and S. Ulam. Über die Permutationsgruppe der natürlichen Zahlenfolge. Stud. Math., 4:134-141, 1933.

[17] Á. Szendrei. A survey of clones closed under conjugation. preprint.

Algebra, TU Wien, Wiedner Hauptstrasse 8-10/104, A-1040 Wien, Austria

E-mail address: marula@gmx.at

$U R L:$ http://www.dmg.tuwien.ac .at 